\title{
Numerical Simulation of Vapor-Liquid Phase Flow and Vapor-Liquid Phase Change
}

\author{
Y.L Huang, S.S. Shi \\ School of Mechanical and Automotive Engineering \\ South China University of Technology \\ Guangdong, China
}

\begin{abstract}
By using the VOF method to calculate free surface and CSF model to treat surface tension, the numerical simulation of the rising bubble in water was accomplished. The results show that the bubble transforms and rotates during rising in the water, and its trajectory changes from straight to harmonic motion with its velocity increasing. The phenomenon coincides well with the experimental observation. The evaporation and condensation processes were also simulated by programming the user-defined function (UDF) as source terms of the heat and mass transfer equations, and a case study was carried out to verify the feasibility of the mathematical model presented to simulate the vapour-liquid phase change process.
\end{abstract}

Keywords-vapor-liquid phase flow; vapor-liquid phase change; mass transfer; heat transfer

\section{INTRODUCTION}

The vapor-liquid phase flow and vapor-liquid phase change are both usually found in nature and are widely used in chemical and energy applications such as reactors, steam generators and cooling systems. Presently, the two-phase flow has found applications in micro-electro-mechanical systems, medical systems and bioengineering systems. Hence, the knowledge of fluid mechanics and heat transfer in micro-scale devices is necessary to improve their efficiencies, which leads to the study of the two phase flow in micro channels with an emphasis on void fraction, pressure drop, flow pattern, and the heat transfer coefficient. These are important elements for the design and development of devices in order to provide the best performance. Phase change problems are encountered in various applications such as thermal energy storage, porous heat pipes, enhancement of condensation, and geothermal systems. Comprehensive reviews on this subject are also available in the literature [1].

From what we discussed above, the studies on vapor-liquid phase flow and vapor-liquid phase change which lead to fully and deeply understanding of the processes are necessary and important. In the past, there are mainly two methods for studying them which are so-called experiment method and the lumped parameter method [2]. The lumped parameter method is actually one based on calculation which has the advantages of simplicity and high efficiency. However, this method is not appreciated by researchers because it cannot get the details of the physical processes occurring in devices. The numerical simulation can be used to quantitatively study the physical phenomena concerning to the thermal performance and visually reflect what happen in heat pipes. Up to date, the method of simulation is obviously the most effective way to research the two phase flow and two phase change.

This paper presents the mathematical model and the computational methods for two cases containing vapour-liquid flow and vapour-liquid phase change, which would be further developed to modelling and simulation of heat pipes therein occurring the evaporation and condensation processes and complex two phase flow.

\section{THE MATHEMATICAL MODEL AND NUMERICAL SOLVING METHOD}

Based on VOF model in FLUENT software, we studied vapor-liquid phase flow and vapor-liquid phase change by simulating the process of bubble rising in water and the evaporation and condensation of water in an upside-down Ushape pipe.

A. VOF Model

VOF multiphase model [3] mainly solves a set of momentum equations, and tracks each fluid's volume fraction in the computational domain at the same time, so as to simulate multiphase flow. VOF model is a fixed Eulerian mesh surface tracking method.

If the volume fraction of the qth phase in the unit is represented as $\alpha \mathrm{q}$, there are three cases as follow:

$\alpha q=0$ : The unit has no q-phase fluid;

$\alpha q=1:$ The unit is full of q-phase fluid;

$0<\alpha \mathrm{q}<1$ : The unit contains q-phase fluid and other fluid.

By solving the following values of volume fraction of continuity equation, $\alpha \mathrm{q}$ is obtained:

$$
\frac{\partial}{\partial t}\left(\alpha_{q} \rho_{q}\right)+\nabla \cdot\left(\alpha_{q} \rho_{q} \overrightarrow{v_{q}}\right)=S_{\alpha q}+\sum_{p=1}^{n}\left(m_{p q}-m_{q p}\right)
$$

where, mpq is the mass transfer from $\mathrm{p}$ phase to q phase; mqp is the mass transfer from $q$ phase to $p$ phase. By default, the source term $\mathrm{S} \alpha \mathrm{q}$ at the right side of the equation is zero, but it can be specified as a non-zero constant.

\section{B. Vapor-Liquid Phase Flow Model and the Equations}

In this paper, the rising air bubble in water is simulated. Surface tension at vapor-liquid interface was solved by using CSF model [4]. Only the force perpendicular to the interface was considered, and the surface tension along the surface is 
constant. It's easy to prove that, the pressure on the whole surface depends on the surface tension coefficient $(\sigma)$ and the two radii (R1 and R2) of curvature of orthogonal on the surface:

$$
p_{1}-p_{2}=\sigma\left(\frac{1}{R_{1}}+\frac{1}{R_{2}}\right)
$$

where, $\mathrm{p} 1$ and $\mathrm{p} 2$ are the pressure of the two phases on each side of the interface, respectively. Surface curvature is calculated by gradient from the surface.

Whether considering the effect of surface tension is determined by two dimensionless numbers: Reynolds number $(\mathrm{Re})$ and capillary number $(\mathrm{Ca})$ for the situation of $\operatorname{Re}<<1$, or Reynolds number and Weber number (We) for Re $>>1$.

We can calculate these numbers by the following equations:

$$
R e=\frac{\rho \cdot u_{b} \cdot d}{\mu}, \quad C a=\frac{\mu \cdot u_{b}}{\sigma}, \quad W e=\frac{\rho L u_{b}{ }^{2}}{\sigma}
$$

where, $\sigma$ is surface tension $(\mathrm{N} / \mathrm{m}), \rho$ is fluid density, $\mu \mathrm{b}$ is free flow speed, $\mathrm{L}$ is the characteristic length.

The effect of surface tension is notable when $\mathrm{Ca}$ or $\mathrm{We}$ is small. When $\mathrm{Ca} \gg>1$ or $\mathrm{We}>>1$, the effect of surface tension can be ignored. In this model, $\mathrm{Re}=492>>1$, and we get $\mathrm{We}=1.08 \approx 1$, so the influence of surface tension must be taken into account.

\section{Vapor-Liquid Phase Change Model}

In the process of evaporation and condensation, many equations of mass transfer are proposed according to the principle of gas dynamics. Among them, Hertz-Knudsen equation [5] is used to calculate the mass flow through the vapor-liquid phase interface. The form is shown as following:

$$
J_{\mathrm{o}}=\alpha_{c} \frac{\sqrt{M}}{\sqrt{2 \pi R}}\left(\frac{p}{\sqrt{T_{v}}}-\frac{p_{s a t}\left(T_{l}\right)}{\sqrt{T_{l}}}\right)
$$

where Jo is the mass flow through the vapor--liquid interface $(\mathrm{kg} / \mathrm{m} 2 \cdot \mathrm{s}) . \mathrm{M}$ is molar mass. $\mathrm{R}$ is universal gas constant. $\mathrm{Tv}$ and $\mathrm{Tl}$ are the temperatures of vapor and liquid, respectively. $\mathrm{p}$ and psat are pressure and saturation pressure, respectively. $\alpha \mathrm{c}$ is adjustment coefficient.

Clausius-Clapeyron equation associates the pressure and temperature in saturated state:

$$
\frac{d p}{d T}=\frac{\Delta H}{T\left(1 / \rho_{v}-1 / \rho_{l}\right)}
$$

where, $\Delta H$ is the latent heat of vaporization.

When the pressure and temperature is close to saturation, Eq. (5) can be transformed into Eq. (6). Then take Eq. (6) into Eq. (4), we obtain Eq. (7)

$$
\begin{gathered}
p-p_{\text {sat }}=-\frac{\Delta H}{T\left(1 / \rho_{v}-1 / \rho_{l}\right)}\left(T-T_{\text {sat }}\right) \\
J_{o}=\alpha_{c} \frac{\sqrt{M}}{\sqrt{2 \pi R T_{\text {sat }}}} \frac{\Delta H}{\left(1 / \rho_{v}-1 / \rho_{l}\right)} \frac{T_{\text {sat }}-T}{T_{\text {sat }}}
\end{gathered}
$$

Mass transfer in phase change is added to the mass conservation equation as a source term with the unit of $\mathrm{kg} /(\mathrm{m} 3 \cdot \mathrm{s})$. Multiplication of Eq. (7) with the volumetric interfacial surface area $\alpha i$ gives the mass flux with the correct units. Thus, the mass flux through the vapor-liquid interface is changed into the following form:

$$
J=\alpha_{i} \alpha_{\mathrm{c}} \frac{\sqrt{M}}{\sqrt{2 \pi R T_{\mathrm{sat}}}} \frac{\Delta H}{\left(1 / \rho_{v}-1 / \rho_{l}\right)} \frac{T_{\text {sat }}-T}{T_{\text {sat }}}
$$

\section{ANALYSES OF SIMULATION RESULTS}

\section{A. Vapor-Liquid Phase Flow Simulation Results}

Several typical patterns of the bubble movement at different moments are shown in Figure 1, of which the red represents air and the blue for water.
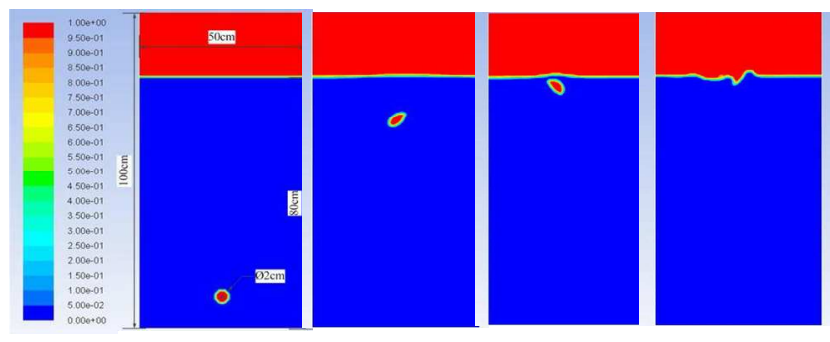

a) $\mathrm{t}=0 \mathrm{~s} \quad$ b) $\mathrm{t}=0.3655 \mathrm{~s} \quad$ c) $\mathrm{t}=0.4687 \mathrm{~s} \quad$ d) $\mathrm{t}=0.4984 \mathrm{~s}$

FIGURE I. BUBBLE SHAPE AND MOVEMENT PATTERNS AT DIFFERENT TIME

The dimension of the model is given in figure 1 (a), and the initial state is set as circular and static as shown. From figure 1 (b) and figure 1 (c) we can see that the bubble deforms and rotates in the rising process, as it is stressed by the water pressure and increased resistance, and the increasing velocity of bubble movement, the changing pressure of surrounding water and the interaction of the bubble and water change its path. As is shown in figure 1 (c), when the bubble is about to arrive the fluid level, it has already impacts on the surface. Figure 1 (d) shows that the bubble has fully overflowed and ruptured with causing slight wave. The whole rising process takes $0.4984 \mathrm{~s}$ and covers a vertical distance of $70 \mathrm{~mm}$. The average speed of air bubble rising is about 140.45 $\mathrm{mm} / \mathrm{s}$.

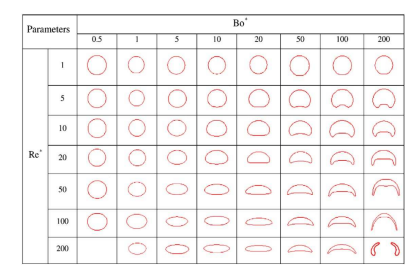

FIGURE II. BUBBLE SHAPE[6]. 


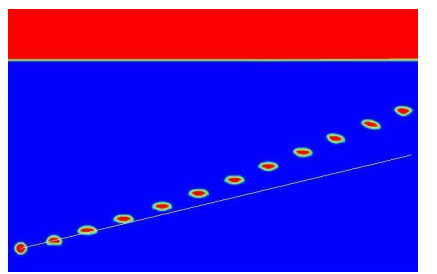

FIGURE III. BUBBLE VERTICAL DISPLACEMENT .

As is shown in figure 2, the bubble shape is mainly influenced by the Reynolds number (Re) and the Bond number (Bo) [6], which is a dimensionless number expressing the ratio of gravitational forces to surface tension forces, when the vapor-liquid density ratio and the viscosity ratio are certain. The Reynolds number is related to the velocity. In other words, the Reynolds number will increase when the velocity of bubble increasing. According to figure 2, bubble shape tends to change from a circular to meniscus, which demonstrates our simulation results.

As is shown in figure 3, we tracked the shape and position of the buble for every $0.03 \mathrm{~s}$ from initial time to $0.33 \mathrm{~s}$. By the help of reference line in the figure, we can see clearly that the bubble is in the accelerated motion at the initial stage. The reason is that the bubble movement velocity and the resistance is small at the beginning, and the buoyancy is greater than the resistance. With the bubble velocity incresing, the resistance increases and equals to the buoyancy finally, then the bubble motion would keep a constant speed.

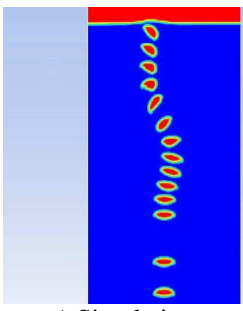

a) Simulation results

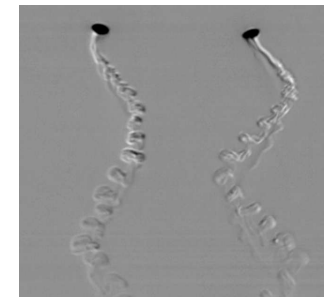

b) Experimental results[7]
FIGURE IV. THE AIR BUBBLE IN HARMONIC MOTION IN WATER.

Figure 4 (a) shows the dynamic process of the bubble rising. As can be seen that the bubble rises along a straight line at the beginning because the bubble is in the moleculer thermal equilibrium state at this stage. It begins left and right swing up at the middle stage of which the bubble keeps similarly a harmonic motion. This is due to the deformed bubble bears ununiform force on its surface. The conclusion has been verified by lots of experiments [7] as shown in figure 4 (b).

\section{B. Vapor-Liquid Phase Change Simulation Results}

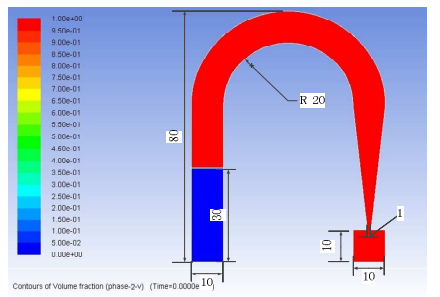

FIGURE V. PHASE CHANGE MODEL.

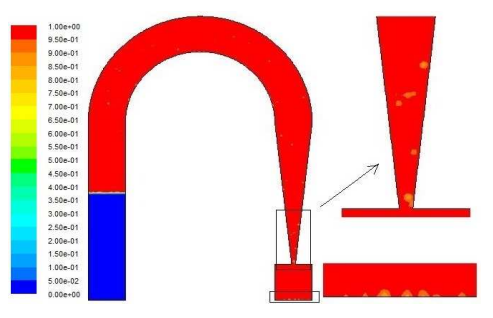

FIGURE VI. THE VAPOR-LIQUID PHASE AT 0.1S.

The sizes of the model (unit: $\mathrm{mm}$ ) and the initial state is shown in figure 5, where the blue part is water, and the red part is water vapor. In order to reduce the computation time, the model is simplified and idealized, which would not weaken the reliability of the model and the algorithm of this paper. We set the left bottom to a constant temperature of $573 \mathrm{~K}$ being an effective heat source. The walls of cooling square at the right are set to the constant temperature of $274 \mathrm{~K}$ being an effective heat sink, and the other sides in the model are set as adiabatic walls. At the initial state, the temperature of each phase and the adiabatic wall is set to $288.15 \mathrm{~K}$.

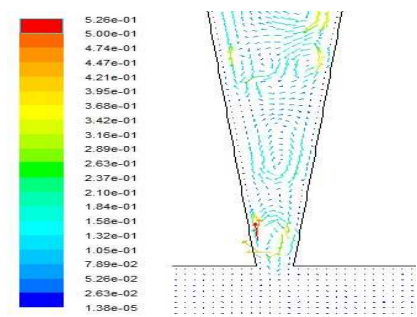

a) Velocity vector at the narrow mouth

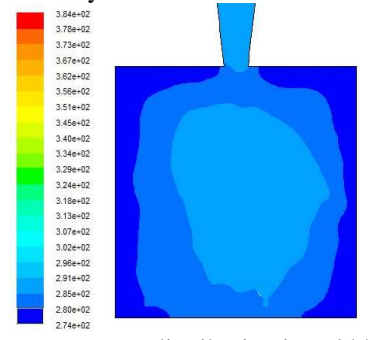

b) Temperature distribution in cold box

FIGURE VII. THE DETAILS OF VAPOR-LIQUID PHASE CHANGE AT $0.1 \mathrm{~S}$.

As is shown in figure 6, at the moment of $0.1 \mathrm{~s}$, some small dispersed droplets appear in the cooling part of the pipe, which means that the vapor condensation has started due to the low initial temperature. Condensation volume mainly concentrated in the narrow mouth $(1 \mathrm{~mm})$ of the right bottom and the $10 \times 10 \mathrm{~mm}$ cold box. According to figure 7 (a), due to the continuity of the flow, the velocity of the vapor phase increases at the right end nozzle to $0.526 \mathrm{~m} / \mathrm{s}$. Figure 7 (b) shows that the vapor temperature is close to the wall temperature of cold box. Obviously, the condensation in the cold box is significant especially near the wall. And because of gravity small drops of condensation are condensed at the bottom of the cold box. 


\section{$\mathrm{n} \cap \mathrm{n} \cap$ \\ a) $\mathrm{t}=0.2 \mathrm{~s}$ \\ FIGURE VIII. \\ b) $\mathrm{t}=0.3 \mathrm{~s}$ \\ c) $\mathrm{t}=0.7 \mathrm{~s}$ \\ d) $\mathrm{t}=1.0 \mathrm{~s}$ \\ VAPOR-LIQUID PHASE CHANGE AT DIFFERENT MOMENTS}

Figure 8 shows the vapor-liquid phase change at different moments. Figure 8 (a) clearly shows that the evaporator has produced a layer of vapor film at $0.2 \mathrm{~s}$, as the wall temperature at the left bottom is as high as $573 \mathrm{~K}$. At the same time, due to the buoyancy the bubble generates tiny disturbance and the surface appears small oscillation. At the bottom of the cold box on the right, the liquid droplets begin to get together. At the bottom of the cold box, the liquid phase gathers together into film.

As shown in Figure 8 (b), at the moment of $0.3 \mathrm{~s}$, as the liquid continuously gets heating from the hot bottom, bubbles are generated at the bottom of left; at the same time, droplets gather in the cold box at the bottom due to gravity. With the time more and more bubbles would be condensed and more and more liquid phase gathers in the cold box.

Figure 8 (c) shows that more bubbles form and move upward due to the buoyancy, with different shapes. The bubbles have been distributed to the most of the liquid phase. Simultaneously more droplets are gathered in the narrow mouth at the right condensation part, and condensed liquid can be obviously seen at bottom of the cold box.

From figure 8 (d), the bubbles have been dispersed throughout the liquid region and cause the free surface fluctuation. More condensed liquid occur at the bottom of cold box. In the phase diagram we captured a droplet falling from the narrow mouth to the bottom.

\section{CONCLUSIONS}

(1) Calculations of Reynolds number and Weber number demonstrate that surface tension must be considered in the simulation of rising bubble in water. The VOF method and CSF model are used to treat the free surface and the surface tension, respectively. The simulation results show that the bubbles deform and rotate during rising in the water. With the velocity increasing, the trajectory of the bubble changes from linear to sinusoidal, which agrees well with the experimental phenomena.

(2) Evaporation and condensation are the most important processes of heat transfer and mass transfer in most applications. Based on the Hertz-Knudsen equation, the control equations of evaporation and condensation are established. And by using the user-defined function (UDF), we complete the numerical simulation of a typical case of vaporliquid phase change, which demonstrates that it is feasible to simultaneously simulate the evaporation and the condensation by using the present model.

\section{ACKNOWLEDGEMENT}

The work was supported in part by the National Natural
Science Foundation of China (No. 51075157).

\section{REFERENCES}

[1] C.Y. Wang \& P. Cheng, Multiphase flow and heat transfer in porous media, Advances in Heat Transfer, vol. 30, , New York, 1997, pp.93-196.

Karthikeyan V.K., Ramachandran K. \& Pallai B.C., Effect of nanofluids on thermal performance of closed loop pulsating heat pipe. Experimental Thermal and Fluid Science, 54:171-178, 2014

[3] Sandra C.K., Geraldine J., Mari G.B.,et al, CFD modeling of all gas.liquid and vapor-liquid flow regimes predicted by the Baker chart. Chemical Engineering Journal, ,138: 349-357,2008

[4] Brackbill J.U., Kothe D.B. \& Zemach C., A continuum method for modelling surface tension. Journal of Computational Physics, 100 (2): 335-354,1992

[5] Lide D.R., CRC handbook of chemistry and physics, Boston: CRC Press, 1998:266

[6] Hua J.S. \& Lou J., Numerical simulation of bubble rising in viscous liquid. Journal of Computational Physics, 222: 769-795,2007

[7] Bian Y.Ch., Dong F., Zhang W.D.,et al, 3D reconstruction of single rising bubble in water using digital image processing and characteristic matrix. Particuology, 11(2): 170-183,2013 